\title{
The use of laser speckle contrast imaging to predict flap necrosis: An experimental study in a porcine flap model
}

Johan Zötterman, Erik Tesselaar and Simon Farnebo

The self-archived postprint version of this journal article is available at Linköping University Institutional Repository (DiVA):

http://urn.kb.se/resolve?urn=urn:nbn:se:liu:diva-156911

N.B.: When citing this work, cite the original publication.

Zötterman, J., Tesselaar, E., Farnebo, S., (2019), The use of laser speckle contrast imaging to predict flap necrosis: An experimental study in a porcine flap model, Journal of Plastic, Reconstructive \& Aesthetic Surgery, 72(5), 771-777. https://doi.org/10.1016/j.bjps.2018.11.021

Original publication available at:

https://doi.org/10.1016/j.bjps.2018.11.021

Copyright: Elsevier

http://www.elsevier.com/

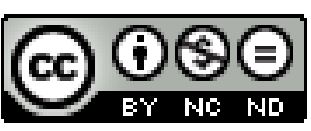




\section{The Use of Laser Speckle Contrast Imaging to predict flap necrosis - an experimental study in a porcine flap model}

Johan Zötterman $^{1}$, Erik Tesselaar ${ }^{2}$, and Simon Farnebo ${ }^{1}$

1. Department of Hand and Plastic Surgery and Burns and Department of Clinical and Experimental Medicine, Linköping University, Linköping, Sweden.

2. Department of Radiation Physics and Department of Clinical and Experimental Medicine,

Linköping University, Linköping, Sweden.

\section{Corresponding author:}

Johan Zötterman

Department of Clinical and Experimental Medicine

Linköping University

58185 Linköping, Sweden

tel. +46703370524

E-mail.johan.zotterman@liu.se 


\section{Abstract}

\section{Background:}

We have evaluated the use of Laser Speckle Contrast Imaging (LSCI) in the perioperative planning in reconstructive surgery. The aim of the study was to investigate if LSCI can predict regions with a high risk of developing postoperative necrosis. Our hypothesis was that, perioperatively, such regions have perfusion values below a threshold value and show a negative perfusion trend.

\section{Methods:}

A porcine flap model based on the cranial gluteal artery perforator was used. Images were acquired prior to surgery, immediately after surgery $(\mathrm{t}=0)$, after 30 minutes $(\mathrm{t}=30 \mathrm{~min})$ and after 72 hours $(\mathrm{t}=72$ h). Regions of Interest (ROI) were chosen along the central axis of the flap. Clinical evaluation of the flap was done during each time point.

\section{Results:}

At $\mathrm{t}=72 \mathrm{~h}$ a demarcation line could be seen at $15.8 \pm 0.4 \mathrm{~cm}$ from the proximal border of the flaps. At $\mathrm{t}=0$ perfusion decreased gradually from proximal to distal ROI. At $\mathrm{t}=30 \mathrm{~min}$ perfusion was significantly lower in ROI distal to the final demarcation line compared to $t=0$ and every flap these ROI had a perfusion $>25$ PU. At $\mathrm{t}=72$ perfusion in ROI proximal of this line returned to baseline levels, while perfusion in distal ROI remained low.

\section{Conclusions:}

In our model a decrease in perfusion during the first 30 minutes after surgery, and a perfusion $<25 \mathrm{PU}$ at $\mathrm{t}=30 \mathrm{~min}$ was a predictor for tissue morbidity 72 hours after surgery, indicating that LSCI is a promising technique for perioperative monitoring in reconstructive surgery. 


\section{Keywords:}

reconstructive surgery; laser speckle contrast imaging; partial flap necrosis

\section{Introduction}

When tissue reconstruction is done using either microvascular free flaps, random flaps, propeller flaps or pedicled flaps, there is always a risk of tissue morbidity that is much dependent on the tissue relation to the feeding vessels. Clinical judgment of tissue viability by the surgeon, on the operation table, remains the golden standard; however, clinical judgment is subjective, dependent on clinical experience and not always reliable for assessment of flap perfusion and long term viability.

Postoperative loss of tissue viability due to compromised circulation may result in complete flap loss or additional revision procedures. This leads to increased risk for infections, poor wound healing and fat necrosis. This will affect both the functional and aesthetic outcome of the surgery.

New non-invasive and minimally invasive technical advancements have been adopted in reconstructive surgery with the aim to improve pre-, peri- and postoperative tissue monitoring. ${ }^{1}$ Despite this, clinical judgment by the surgeon remains the golden standard for perioperative evaluation of the tissue on the operating table. However, clinical judgment is 
subjective, dependent on clinical experience and not always reliable for assessment of flap perfusion and long term viability.

In this experimental study, we evaluated Laser Speckle Contrast Imaging (LSCI) as an alternative method for perioperative assessment in reconstructive surgery.

LSCI is a laser-based technology that, unlike the much slower laser Doppler imager (LDI), allows for a very fast (fraction of a second) assessment of an area of tissue, with a high spatial resolution. ${ }^{3,4}$ LSCI has been used in a number of studies to assess superficial perfusion in surgical flaps. For example, Ngyen et al and Sheikh et al studied blood perfusion in porcine eyelid flaps using LSCI, laser Doppler velocimetry, and thermography and Du et al used LSCI to assess microcirculation in skin flaps in rats. ${ }^{5-8}$

In a previous study, we have shown that LSCI is able to detect postoperative partial and full venous and arterial obstruction. ${ }^{7}$ One of our conclusions was that even if LSCI has a lower variability in detecting changes in venous blood flow compared to laser Doppler flowmetry, the current LSCI systems might be better suited for perioperative assessment than for continuous long term post-operative measurements. ${ }^{9}$

The aim of this study was to investigate if LSCI can be used perioperatively to identify flap areas with compromised circulation, and thereby predict areas with a high risk of postoperative necrosis. Our hypothesis was that a decrease in perfusion could be seen in areas with compromised circulation during the first 30 minutes after the flap was raised. Also, we hypothesized that threshold values of the perfusion could be identified, which may potentially be used perioperatively to assist in planning the flap and for safe removal of tissue parts that would have a poor viability postoperatively.

A porcine pedicled flap model based on the cranial gluteal artery perforator was used for the in this study. A similar model was used by our group in the previously mentioned study to show the usefulness of LSCI for postoperative flap monitoring compared to laser 
Doppler flowmetry. ${ }^{9}$ In this former study, in which the flaps were designed as $12 \mathrm{~cm}$ by 15 $\mathrm{cm}$ rectangles, we could see signs stasis in the most distal parts indicating threatening ischemia. Therefore, in the current study, the flap was elongated to $20 \mathrm{~cm}$ and narrowed to 10 $\mathrm{cm}$ to promote that the most distal part of the flap would be non-viable after three days. The model simulates partial necrosis which is a known complication in reconstructive surgery. ${ }^{10}$

\section{Materials and Methods}

\section{Animals}

Six mixed breed pigs (mean age 4 months, $45 \mathrm{~kg}$, Swedish Landrace pigs) were used in the study.

\section{Surgery}

The pigs were pre-anesthetized with Dexdomitor $0.1 \mathrm{mg} / \mathrm{kg}$, Zoletil $5 \mathrm{mg} / \mathrm{kg}$ and atropine $0.05 \mathrm{mg} / \mathrm{kg}$. Anaesthesia was maintained with Pentobarbital sodium $8 \mathrm{mg} / \mathrm{kg} / \mathrm{h}$ and fentanyl $0.5 \mu \mathrm{g} / \mathrm{kg} / \mathrm{h}$ dissolved in Ringer's acetate given continuously intravenously with motorized infuser along with crystalloid fluids (Ringer acetate). Body temperature, blood pressure, heart rate and oxygen saturation were monitored during the whole procedure.

A $10 \times 20 \mathrm{~cm}$ fascio-cutaneous island flap based on the cranial gluteal artery perforator (CGAP) was raised from the right buttock of each pig. The flap was dissected along the surface of the muscle, including skin, subcutaneous tissue and muscle fascia. The pedicle containing the perforator artery along with comitant veins was isolated (Fig. 1). The flap was then reinserted in its original position using surgical staples. No barrier was inserted between the flap and the underlying muscle. After the initial measurements the flaps were 
dressed in dry swabs and Tegaderm (3M, St. Paul, Minneapolis, USA) and the animal was returned to its box and awakened from anesthesia.

After $72 \mathrm{~h}$ the animal was anesthetized in the same manner as previously described. After the final measurements were done the animal was euthanized with an overdose of pentobarbital sodium and 70\% ethanol without regaining consciousness.

Fig 1: The cranial gluteal artery perforator flap (CGAP). The flap was dissected along the surface of the muscle, including skin, subcutaneous tissue and muscle fascia. The pedicle containing the artery along with comitant veins was isolated.

\section{Clinical Evaluation}

The visual appearance and capillary refill of the flaps were evaluated by two clinically experienced surgeons (authors SF and JZ) at $\mathrm{t}=0 \mathrm{~min}, \mathrm{t}=30 \mathrm{~min}$ and $\mathrm{t}=72 \mathrm{~h}$. This approach was adopted to mimic the clinical setting, where the surgeon constantly evaluates the color and capillary refill of the flap.

\section{Equipment}

A Laser Speckle Contrast Imager (PeriCam PSI System, Perimed AB, Järfälla, Sweden) was used for all measurements. In short, LSCI measures perfusion illuminating the tissue with a divergent laser beam with a wavelength of $785 \mathrm{~nm}$. This creates a speckle pattern over the illuminated area. A monochrome CMOS camera captures the speckle image and the perfusion is calculated in every image pixel by measuring the local speckle contrast. A separate camera captures a color image of the measured area. The principle of the measurement technique of LSCI has been previously described in detail., ${ }^{3,11}$ 


\section{Measurements}

After the pig was anesthetized the area for the flap was marked on the buttock of the pig. Room temperature and vital parameters, including blood pressure and heart frequency, were continuously monitored. The distance between the LSCI camera and the skin was kept between 20 and $25 \mathrm{~cm}$. The settings were the same for all measurements with an acquisition rate of 21 images/s and averaging of the perfusion data from 42 consecutive images, resulting in a total measurement time of $2 \mathrm{~s}$ for each image. The image size was set to correspond to the size of the flap and the spatial resolution of the perfusion image is $0.2 \mathrm{~mm} / \mathrm{pixel}$ at a measurement distance of $20-25 \mathrm{~cm}$. Baseline perfusion of the flap was measured during anesthesia, prior to surgery. Immediately $(\mathrm{t}=0)$, as well as 30 minutes $(\mathrm{t}=30 \mathrm{~min})$ after the flap was raised and reinserted, perfusion was measured with the same settings. After 72 hours $(t=72 \mathrm{~h})$ a final perfusion measurement was made with the same settings as before. Clinical evaluation of the flaps was done at $t=0, t=30$ min and $t=72 \mathrm{~h}$. Digital photographs were taken of the flaps at the respective time points, and demarcation lines were overlaid on each image to indicate where the surgeons believed that viability was questionable. In each flap, the distance from the pedicle base to the demarcation line was measured using Image J software. ${ }^{12}$

\section{Data Analysis}

Perfusion values are presented as PU (Perfusion Units), which is an arbitrary unit calculated from the speckle contrast analysis and is proportional to the concentration and the average velocity of the red blood cells in the measured tissue. ${ }^{3,4}$ All perfusion images were analyzed using the system's analysis software (PSIWin, Perimed, Järfälla, Sweden). Ten 
regions of interest (ROI), two centimeters apart, were chosen from the LSCI images along the central axis of the flap from the proximal to the distal border.

All data is in text and tables are presented as absolute values or mean (SD). Two-way analyses of variance for repeated measures with Tukey's multiple comparisons test were performed to test whether changes from baseline and differences between $t=0$ and $t=30$ minutes were significant. Statistical calculations were done using GraphPad Prism version 7.0 for Windows (GraphPad Software, San Diego, USA). With all analyses, probabilities of less than 0.05 were accepted as significant.

\section{Results}

Room temperature was stable throughout the protocol. No major changes in blood pressure or heart frequency were registered.

\section{Clinical evaluation}

After the flaps were raised $(\mathrm{t}=0 \mathrm{~min})$ a darker area interpreted as an area with compromised circulation could be seen on all flaps at a mean (SD) distance of $10.7(1.9) \mathrm{cm}$ from the proximal border of the flaps, and beyond. At $\mathrm{t}=30 \mathrm{~min}$ the distance to the area with stasis was $10.1(0.8) \mathrm{cm}$ (Figure 2A-B and Figure 5).

After 72 hours, a clear demarcation line was seen at a distance of $15.8(0.4) \mathrm{cm}$ from the proximal border of the flap (Figure 2C). The area distal of this line had clinical signs typical for ischemia with a dark color without any notable capillary refill or arterial bleeding when punctured with a biopsy punch. 


\section{Changes in flap perfusion}

The mean perfusion of the flaps at baseline, at $\mathrm{t}=0$ and at $\mathrm{t}=30 \mathrm{~min}$ for 10 different regions of interest (ROI) is shown in Figure 3. At baseline, the highest perfusion in the flap was $86 \pm 17 \mathrm{PU}$ (ROI 2) and the lowest perfusion was $68 \pm 15 \mathrm{PU}$ (ROI 9). The perfusion decreased slightly from proximal to the distal regions of the flap (at most $20 \%$ decrease at ROI 9 compared to ROI $1, \mathrm{p}<0.001)$. At $\mathrm{t}=0$, a general decrease in perfusion was observed in all ROI, compared to baseline (ranging from $75.6 \pm 17.8 \mathrm{PU}$ in ROI 1 to $30.8 \pm 5.4 \mathrm{PU}$ in ROI 10). The decrease in perfusion from the proximal to the distal side of the flap became more pronounced with time, with ROI 10 having a 59\% and 70\% decrease in perfusion compared to ROI 1 , at $\mathrm{t}=0$ and $\mathrm{t}=30$ min, respectively $(\mathrm{p}<0.001)$.

Changes in perfusion between baseline and either $\mathrm{t}=0$ or $\mathrm{t}=30$ min were significant in all ROI $(\mathrm{p}<0.001)$. Between $\mathrm{t}=0$ and $\mathrm{t}=30 \mathrm{~min}$, a significant decrease in perfusion could only be seen in the distal parts of the flap (ROI $8-10, p=0.03)$. In the proximal and medial ROI, perfusion either increased or was stable during the first 30 minutes after raising the flap (Figure 2F).

At $\mathrm{t}=30 \mathrm{~min}$, no ROI distal to the demarcation line at $\mathrm{t}=72 \mathrm{~h}$ had a perfusion above 25 PU (Table 1).

At $t=72 \mathrm{~h}$, perfusion in the area proximal to the demarcation line (ROI 1-8) had recovered to values not significantly different from baseline. In ROI 9 perfusion increased to a value not significantly different from $t=0$, and in ROI 10 the perfusion remained on a value not significantly different from $t=30$ minutes. 
Fig 2: Example of changes in visual appearance (A-C) and perfusion (D-F) in a CGAP flap, directly (A, D) and 30 minutes (B, E) after raising the flap. The dashed black line represents the proximal border of the area with compromised circulation as predicted by clinical assessment. The dashed white line (C) represents the proximal border of the manifest ischemic area at $\mathrm{t}=72$ hours. The colored dots indicate the regions of interest (ROI) in which perfusion was measured. ROI are numbered 110 , starting left to right (proximal to distal). (F) shows the change in perfusion from $\mathrm{t}=0$ to $\mathrm{t}=30 \mathrm{~min}$ in the different ROI (green=viable, yellow intermediate and red ischemic) in the same flap as A-E.

Fig 3: The mean (SD) perfusion in 10 different regions of interest (ROI) of the flaps (proximal to distal) at three different time points; baseline (before surgery), directly after raising the flap ( $\mathrm{t}=0 \mathrm{~min})$ and 30 minutes after raising the flap $(\mathrm{t}=30$ min). Changes in perfusion between baseline and either $\mathrm{t}=0$ or $\mathrm{t}=30$ min were significant in all $\mathrm{ROI}(\mathrm{p}<0.001)$. Between $\mathrm{t}=$ 0 and $t=30 \mathrm{~min}$, a significant decrease in perfusion could only be seen in the distal ROI $(8-10, p=0.03)$.

\section{ROI Flap 1 Flap 2 Flap 3 Flap 4 Flap 5 Flap 6 Mean(SD)}

\begin{tabular}{lllllllll}
\hline $\mathbf{1}$ & 85.2 & 55.0 & 57.4 & 104.6 & 40.9 & 71.2 & $69.0(23.0)$ \\
$\mathbf{2}$ & 84.9 & 60.5 & 50.5 & 91.4 & 41.7 & 66.4 & $65.9(19.3)$ \\
$\mathbf{3}$ & 78.9 & 57.8 & 46.5 & 68.7 & 44.2 & 67.1 & $60.5(13.5)$ \\
$\mathbf{4}$ & 68.5 & 55.2 & 43.6 & 59.4 & 42.5 & 56.7 & $54.3(9.9)$ \\
& & & & & & & \\
$\mathbf{5}$ & 59.3 & 49.3 & 34.0 & 48.7 & 37.6 & 46.2 & $45.8(9.0)$
\end{tabular}




$\begin{array}{lrrrrrrr}\mathbf{6} & 50.4 & 45.2 & 32.7 & 32.6 & 32.5 & 36.0 & 38.2(7.7) \\ \mathbf{7} & 34.5 & 30.3 & 31.8 & 26.4 & 26.5 & 27.4 & 29.5(3.3) \\ \mathbf{8} & 25.3 & 22.3^{*} & 26.4 & 26.8 & 23.7 & 19.2 & 24.0(2.9) \\ \mathbf{9} & 21.4^{*} & 21.2^{*} & 20.6^{*} & 24.6^{*} & 21.2^{*} & 15.5^{*} & 20.7(2.9) \\ \mathbf{1 0} & 23.2^{*} & 23.0^{*} & 20.9^{*} & 24.1^{*} & 19.4^{*} & 15.4^{*} & 21.0(3.3)\end{array}$

Table 1: Perfusion (PU) in the different ROI in the six flaps at $\mathrm{t}=30 \mathrm{~min} . *$ represent $\mathrm{ROI}$ from areas distal to the demarcation line at $\mathrm{t}=72$ hours. Note that there is no ROI with perfusion above $25 \mathrm{PU}$ at $\mathrm{t}=30 \mathrm{~min}$ in areas distal to the demarcation line at $\mathrm{t}=72$ hours.

\section{Discussion}

Partial flap necrosis is a well described problem in reconstructive surgery. In a metaanalysis from 2016, Xiong et al reported 6\% partial necrosis in flaps for reconstruction of defects in lower extremities. ${ }^{13}$ In 2013, Lie et al reviewed 98 articles that reported DIEP flaps that experienced partial necrosis. The reported rate of partial flap necrosis in these articles ranged from 0 to $11 \% .{ }^{10}$ The authors concluded that since there is no consensus around the classification or definition of partial necrosis, it is hard to tell how frequent the problem is. Still, the condition undoubtedly causes great discomfort for the patient and any means to reduce the complication rates further would be beneficial, both in terms of shortened length of stay in hospital and reduced healthcare costs. A tool that could be used as a reliable indicator of flap areas with compromised circulation would be a valuable contribution in the ongoing efforts to improve surgical outcome. 
One method for perioperative imaging of tissue viability that has gained ground in recent years is fluorescence angiography (FA), a vascular imaging method that combines indocyanine green (ICG) with laser angiography to visually assess superficial blood flow. The dye, ICG, has an excellent safety profile, but has to be administered intravenously for each assessment and the cost per case is relatively high. ${ }^{14,15}$

In our study we have used the LSCI as a tool to predict partial flap necrosis in a porcine fasciocutaneous flap model. LSCI has some clear advantages compared to FA for perioperative evaluation of tissue viability. It is a completely noninvasive method, with no need for dye injection prior to assessment, which means that the cost per case is negligible after the initial investment. This also implies that the measurements can be made repeatedly during the operation, and follow-up measurements can be done during the postoperative phase, in case the surgeons suspect that there may be problems with vessel patency or clot formation.

Two of the authors of this study have many years of experience of microsurgery, burn surgery and replantation surgery, and regularly evaluate tissue viability in a clinical setting. Nevertheless, based on visual appearance and capillary refill during the first 30 minutes after the flap was raised, these observers typically overestimated the areas that would present clinical signs of ischemia three days after the surgery.

It is well known that microcirculation at skin regions further away from the main supplying vessels, in this case the CGAP, will display lower perfusion values ${ }^{16}$. In the pig buttock, one may expect there to be multiple supplying vessels that supply the skin segment before raise of the flap, but in this case it seems likely that regions closer to CGAP will be better perfused, even before the flap is raised. Indeed, this is in agreement with our findings, i.e. even before raising the flap, we observed a decreasing perfusion from proximal to distal areas of the flap. 
During the first 30 minutes after the flap was raised, perfusion in the distal parts of the flaps (ROI 8-10) decreased significantly, while perfusion in the more proximal ROI did not decrease or even, in some flaps, increased during the same time period. Importantly, the areas in which perfusion decreased during the first 30 minutes showed clear clinical signs of ischemia three days after the surgery.

Directly after the flaps were raised, all ROI distal to the final demarcation line had a perfusion below $40 \mathrm{PU}$. The variation of the perfusion in the distal parts of the flaps at this time point was large, and in five of the flaps, areas with initial perfusion between 30 and 40 PU recovered and were viable at the end of the study.

After 30 minutes, the perfusion in all ROI distal to the final demarcation line was below 25 PU. At this time point, there was also a smaller variation in perfusion in the distal ROI between the different flaps. This indicates that LSCI assessment of free flap viability should be done for at least thirty minutes after the flap is raised and that perfusion values below 25 PU after 30 minutes are an indicator of compromised circulation and thus could be considered a threshold value, at least in our material. However, the perfusion in flap 5 and 6 was below 25 PU 30 minutes after the flaps were raised in areas proximal to the final demarcation line. This means that if a threshold value of 25 PU had been used as the only indicator of threatening ischemia in a clinical setting, there would have been a risk of unnecessary wide excisions. If the threshold value had been set to $22 \mathrm{PU}$, there would not have been any unnecessary excisions in our material. Instead, later ischemic areas would have been missed in flap 2 and 6 . Thus, although a decrease in perfusion during the first thirty minutes after the flap is raised was found to be a strong predictor for tissue morbidity, other factors, such as the initial perfusion value and clinical appearance should likely be used in conjunction with the LSCI evaluation to guide surgical decisions. 
As a clinical example, a perfusion image, as acquired using LSCI, of a radial forearm flap (for phalloplasty) is shown in Figure 4. Twenty minutes after the flap was raised, an area with low perfusion (around $25 \mathrm{PU}$ ) was identified at the edge of the flap. This area was considered non-viable on the third postoperative day and was subsequently resected. It is interesting to note that the perfusion values in the non-viable part of the flap are in the same range as non-viable areas in the flap model presented in the current study.

Figure 4. Clinical example of a radial forearm flap for phalloplasty 20 minutes after the flap was raised. The arrow in (A) indicates an area with low perfusion ( $25 \mathrm{PU})$. In the corresponding color image (B), the area with clinical signs of reduced perfusion is marked by the surgeon with a dotted line. The same area was considered non-viable on the $3^{\text {rd }}$ postoperative day and subsequently resected (image courtesy of Professor D'Arpa, University Hospital Gent, Belgium).

In our earlier study we could not see any significant difference in perfusion during the first 30 minutes after the flaps were raised in neither proximal nor distal ROI. ${ }^{7}$ The distal ROI in the previous study, in which shorter flaps were used, correlate to more proximal ROI in the current study (approx. ROI 1-7). Interestingly, in the current study, the perfusion in the proximal ROI (ROI 1-8) had normalized or even increased after three days, compared to preoperative measurements. This strengthens our conclusion that a decrease in perfusion during the first thirty minutes after the flap is raised indicates a compromised circulation and a high risk of developing necrosis.

This study has a number of limitations. As with FA, the surface of the flap has to be exposed to be able to be assessed with LSCI. This means that the technique cannot be used on buried flaps after insertion. Also care should be taken not to extrapolate these results to other 
flap types such as myocutaneous, osteocutaneous or muscle flaps since these may have different perfusion characteristics.

We acknowledge that LSCI assessment of skin perfusion might differ between humans and pigs. There are now a few studies in which LSCI has been used for assessment of microcirculation in pig skin. ${ }^{5,6,9,17,18}$ Even if results from these studies might not be translated directly to clinical conditions, we assume that since porcine skin is fairly similar to the human skin, LSCI will measure similar perfusion levels in both species. ${ }^{19,20}$

We used a model based on a pedicled flap and not a free flap since the aim of this study was to evaluate the possibility of using LSCI to predict ischemic areas without actually being interested in the cause of the ischemia. A free flap model would have made the study set up considerably more complicated without any major contribution to the final results.

In this study, we looked at absolute threshold perfusion values as a measure of risk for later ischemia in different regions of the flap, as this is the most straightforward method to use LSCI in clinical practice. However, it is possible that the decrease in perfusion from baseline is more predictive of risk, and future work should be done to investigate the use of regional perfusion changes as a risk measure. This would however require a baseline measurement for each ROI, and the perfusion in the specific regions would have to be related to their respective baseline perfusion.

We choose not to use an artificial barrier between the flap and the underlying muscle, although this might have affected the viability of the flap at the final assessment due to neovascularization. However, at least one study has shown that the minimal critical period for flap survival for arterial occlusion is six days. ${ }^{21}$ Therefore, we believe that a barrier would not have affected the tissue viability and thereby the visual appearance of the flap three days after surgery. 
Another limitation is the small number of subjects. Six flaps is too few to be able to draw any certain conclusion on which threshold value that should be chosen to keep both sensitivity and specificity of the method high. On the other hand it is doubtful that any threshold value obtained from porcine studies could be translated directly to human conditions. Therefore a large scale animal study could not be ethically motivated, since even with a large number of animals, the obtained threshold value might not be representative for patients. Instead future studies should be directed towards pre-, peri- and postoperative assessment of free flaps using LSCI in a larger cohort of patients.

In conclusion, we have found that a decrease in perfusion during the first 30 minutes after raising the flap, and a perfusion below $25 \mathrm{PU}$ after 30 minutes was a predictor for tissue morbidity 72 hours after surgery in a porcine flap model. These findings suggest that LSCI is a promising technique for perioperative monitoring of flaps and for identifying areas at risk of necrosis, and future studies will hopefully show the clinical usefulness of LSCI in flap surgery. $^{22}$

\section{Acknowledgements:}

We thank Dan Linghammar at the Centre for Teaching and Research in Disaster Medicine and Traumatology, Linköping University Hospital for invaluable assistance with animal care and perioperative assistance.

\section{Conflicts of Interest}


None to declare

\section{Funding}

Funding was provided by County of Östergötland.

\section{Ethical Approval}

Ethical approval was given by the Regional Ethical Committee (Jordbruksverket) registration number 90-15.

\section{References}

1. Smit JM, Zeebregts CJ, Acosta R, Werker PM. Advancements in free flap monitoring in the last decade: a critical review. Plast Reconstr Surg 2010: 125: 177-85.

2. Fitzgerald O'Connor E, Rozen WM, Chowdhry M, et al. Preoperative computed tomography angiography for planning DIEP flap breast reconstruction reduces operative time and overall complications. Gland Surg 2016: 5: 93-8.

3. Briers D, Duncan DD, Hirst E, et al. Laser speckle contrast imaging: theoretical and practical limitations. J Biomed Opt 2013: 18: 066018.

4. Briers JD, Richards G, He XW. Capillary Blood Flow Monitoring Using Laser Speckle Contrast Analysis (LASCA). J Biomed Opt 1999: 4: 164-75.

5. Nguyen CD, Sheikh R, Dahlstrand U, Lindstedt S, Malmsjo M. Investigation of blood perfusion by laser speckle contrast imaging in stretched and rotated skin flaps in a porcine model. J Plast Reconstr Aesthet Surg 2017.

6. Sheikh R, Memarzadeh K, Torbrand C, et al. Blood Perfusion in a Full-Thickness Eyelid Flap, Investigated by Laser Doppler Velocimetry, Laser Speckle Contrast Imaging, and Thermography. Eplasty 2018: 18: e9.

7. Nguyen CD, Hult J, Sheikh R, et al. Blood Perfusion in Human Eyelid Skin Flaps Examined by Laser Speckle Contrast Imaging-Importance of Flap Length and the Use of Diathermy. Ophthal Plast Reconstr Surg 2017.

8. Du Z, Zan T, Li H, Li Q. A study of blood flow dynamics in flap delay using the full-field laser perfusion imager. Microvasc Res 2011: 82: 284-90.

9. Zotterman J, Bergkvist M, Iredahl F, Tesselaar E, Farnebo S. Monitoring of partial and full venous outflow obstruction in a porcine flap model using laser speckle contrast imaging. $J$ Plast Reconstr Aesthet Surg 2016: 69: 936-43.

10. Lie KH, Barker AS, Ashton MW. A classification system for partial and complete DIEP flap necrosis based on a review of 17,096 DIEP flaps in 693 articles including analysis of 152 total flap failures. Plast Reconstr Surg 2013: 132: 1401-8.

11. Draijer M, Hondebrink E, van Leeuwen T, Steenbergen W. Review of laser speckle contrast techniques for visualizing tissue perfusion. Lasers Med Sci 2009: 24: 639-51. 12. Schneider CA, Rasband WS, Eliceiri KW. NIH Image to ImageJ: 25 years of image analysis. Nat Methods 2012: 9: 671-5. 
13. Xiong L, Gazyakan E, Kremer T, et al. Free flaps for reconstruction of soft tissue defects in lower extremity: A meta-analysis on microsurgical outcome and safety. Microsurgery 2016: 36: 511-24.

14. Gurtner GC, Jones GE, Neligan PC, et al. Intraoperative laser angiography using the SPY system: review of the literature and recommendations for use. Ann Surg Innov Res 2013: 7: 1.

15. Lee BT, Matsui A, Hutteman M, et al. Intraoperative near-infrared fluorescence imaging in perforator flap reconstruction: current research and early clinical experience. J Reconstr Microsurg 2010: 26: 59-65.

16. Braverman IM. The cutaneous microcirculation: ultrastructure and microanatomical organization. Microcirculation 1997: 4: 329-40.

17. Sheikh R, Dahlstrand U, Memarzadeh K, et al. Optimal Epinephrine Concentration and Time Delay to Minimize Perfusion in Eyelid Surgery: Measured by Laser-Based Methods and a Novel Form of Extended-Wavelength Diffuse Reflectance Spectroscopy. Ophthal Plast Reconstr Surg 2017.

18. Sheikh R, Memarzadeh K, Torbrand C, Blohme J, Malmsjo M. Hypoperfusion in response to epinephrine in local anaesthetics: Investigation of dependence on epinephrine concentration, spread of hypoperfusion and time to maximal cutaneous vasoconstriction. $\mathrm{J}$ Plast Reconstr Aesthet Surg 2017: 70: 322-29.

19. Debeer S, Le Luduec JB, Kaiserlian D, et al. Comparative histology and immunohistochemistry of porcine versus human skin. Eur J Dermatol 2013: 23: 456-66. 20. Meyer W, Schwarz R, Neurand K. The skin of domestic mammals as a model for the human skin, with special reference to the domestic pig. Curr Probl Dermatol 1978: 7: 39-52. 21. Yoon AP, Jones NF. Critical time for neovascularization/angiogenesis to allow free flap survival after delayed postoperative anastomotic compromise without surgical intervention: A review of the literature. Microsurgery 2016: 36: 604-12.

22. Jansen SM, de Bruin DM, van Berge Henegouwen MI, et al. Can we predict necrosis intra-operatively? Real-time optical quantitative perfusion imaging in surgery: study protocol for a prospective, observational, in vivo pilot study. Pilot Feasibility Stud 2017: 3: 65. 



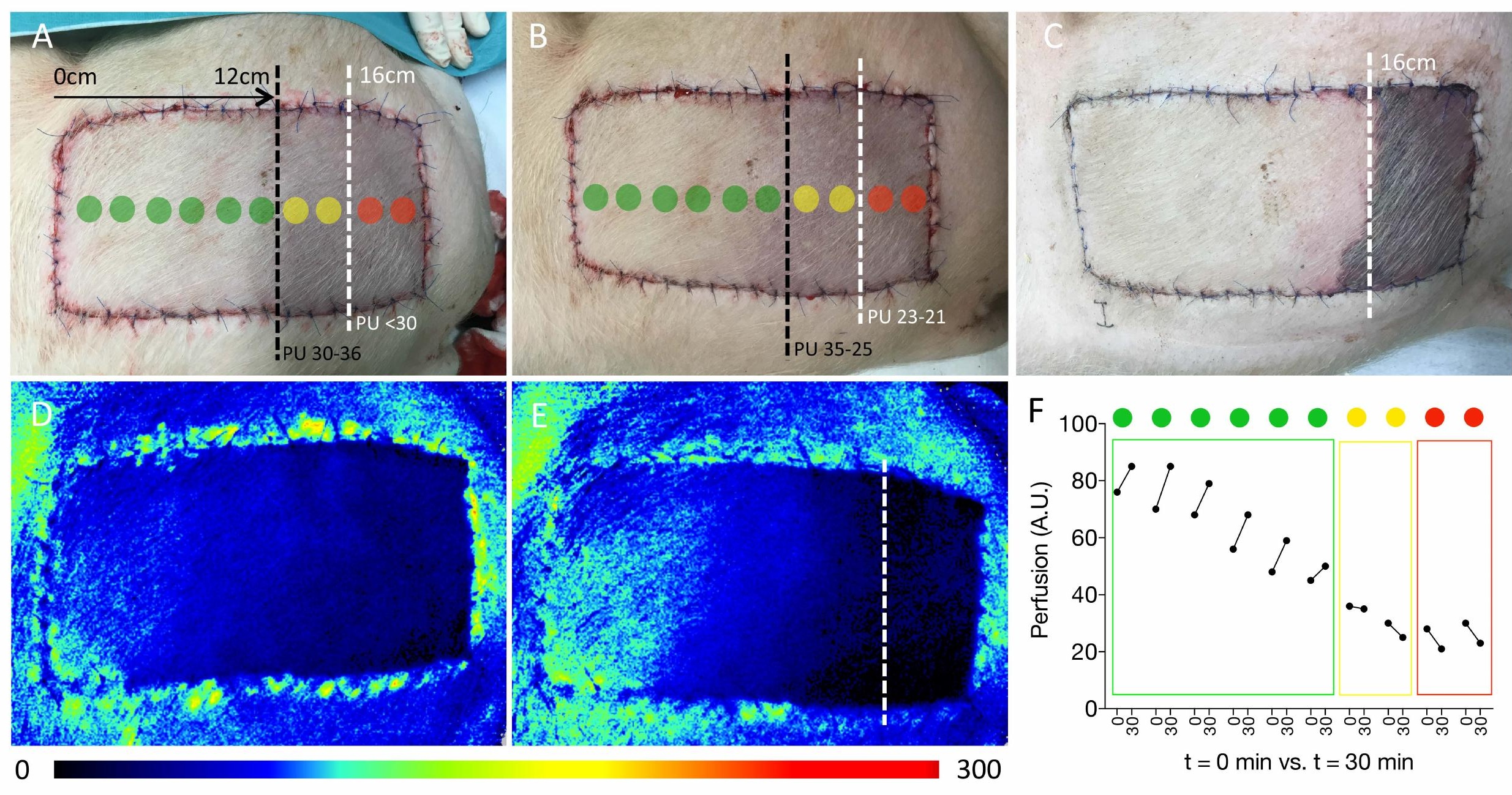


$\rightarrow$ Baseline $\leftarrow t=30$

$$
\rightarrow t=0 \quad \leftrightarrow t=72 \text { hours }
$$

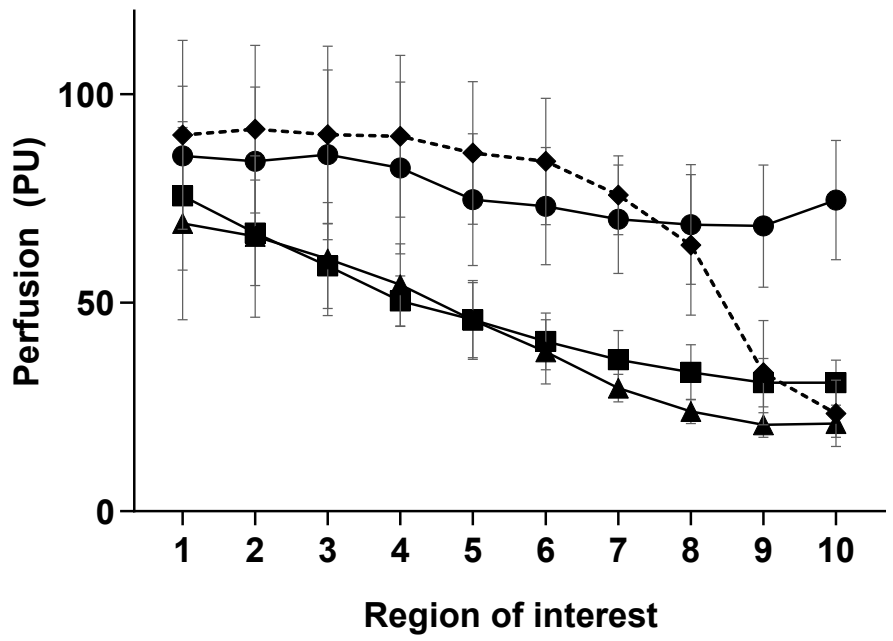




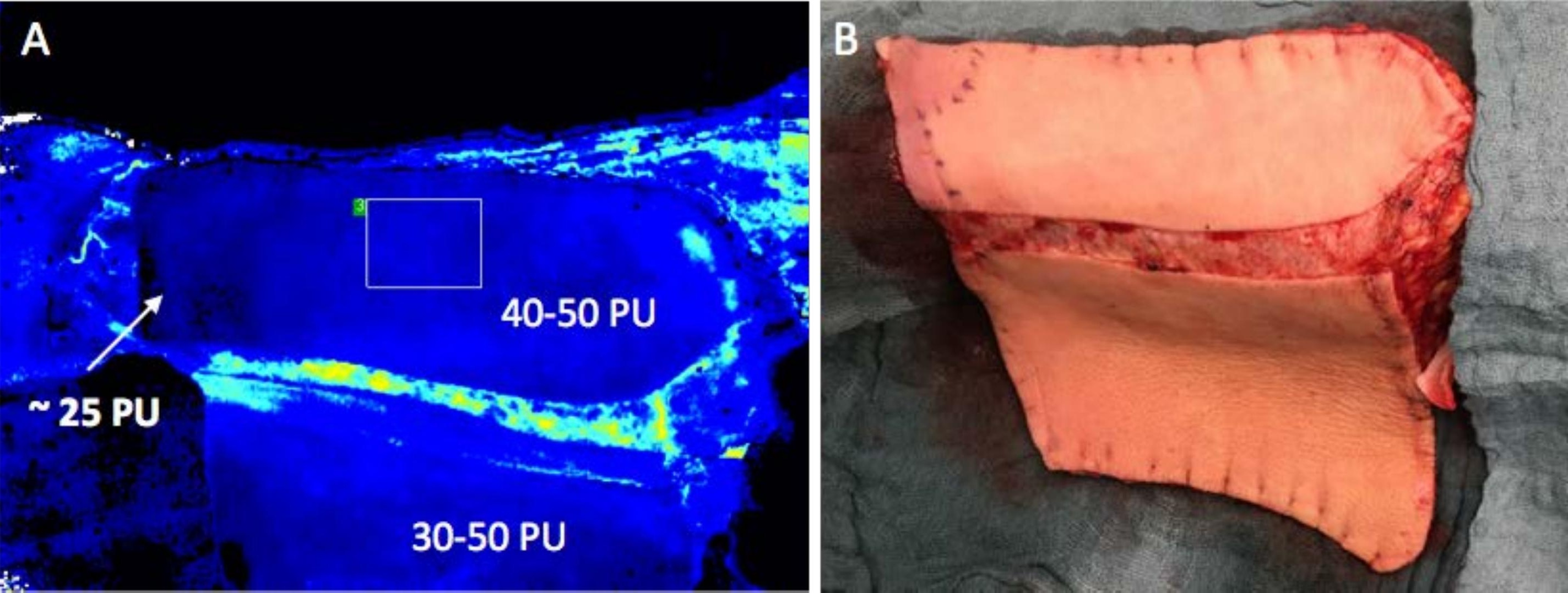

